\title{
Efficient Authentication for Users Autonomy in Next Generation All-IP Networks
}

\author{
Christoforos Ntantogian \\ Department of Informatics and \\ Telecommunications, \\ University of Athens, Greece \\ ntantogian@di.uoa.gr
}

\author{
Christos Xenakis \\ Department of Technology \\ Education and Digital Systems, \\ University of Piraeus, Greece \\ xenakis@unipi.gr
}

\author{
Ioannis Stavrakakis \\ Department of Informatics and \\ Telecommunications, \\ University of Athens, Greece \\ ioannis@di.uoa.gr
}

\begin{abstract}
Next Generation Networks (NGNs) provide multimedia services to mobile users through different access networks that facilitate users autonomy. The security architecture of NGNs specifies that a WLAN user must follow a multi-pass Authentication and Key Agreement (AKA) procedure in order to get access to the IP multimedia subsystem (IMS) services. This paper proposes an improved one-pass AKA procedure for NGNs that reduces significantly the authentication overhead compared to the multipass, without compromising the provided security services. A communication cost analysis is provided that estimates the cost improvement of the proposed one-pass over the multi-pass AKA authentication procedure. The proposed procedure has minimal impact on the network infrastructure and functionality and does not require any changes to the existing authentication protocols.
\end{abstract}

\section{Keywords}

NGN, Users Autonomy, Authentication, EAP-AKA, IKEv2, IMSAKA

\section{INTRODUCTION}

Traditional networks have been constructed and coordinated centrally according to a single plan with a specific management direction. By contrast Next Generation Networks (NGNs) are expected to grow more chaotically in distributed manner with no centrally-mandated goals or levels of service. In such networks service provision is achieved using a choice of different Access Networks (AN) that facilitate users' autonomy and mobility. A prominent AN technology is considered to be Wireless LANs (WLAN) that are capable of supporting multimedia applications such as video conference, video streaming, voice over IP (VoIP), etc. The provision of these multimedia services is relied on the IP Multimedia Subsystem (IMS) [3], which is based on a distributed All-IP network architecture. Although NGNs offer a variety of new service perspectives and communication paradigms, they may also raise new security concerns, mainly, due the heterogeneity of the employed technologies.

To address the security concerns and promote the proliferation of NGNs, a new security architecture is currently under study [2][4] that aims at protecting the mobile users, the data transferred and the underlying network. One of the features of this architecture specifies that a WLAN user must follow a multi-pass Authentication and Key Agreement (AKA) procedure in order to

Permission to make digital or hard copies of all or part of this work for personal or classroom use is granted without fee provided that copies are not made or distributed for profit or commercial advantage and that copies bear this notice and the full citation on the first page. To copy otherwise, or republish, to post on servers or to redistribute to lists, requires prior specific permission and/or a fee.

Bionetics '07, December 10-13, 2007, Budapest, Hungary.

Copyright 2007 ICST 978-963-9799-11-0 get access to the IMS services. This includes three distinct authentication steps (see Figure 1). In the first step, the user executes the (Extensible Authentication Protocol) EAP-AKA protocol [7] that registers him to the WLAN domain. In the second step, the user executes the Internet Key Exchange version 2 (IKEv2) protocol [8] that encapsulates EAP-AKA, which registers him to the $3 \mathrm{G}$ public land mobile network (PLMN) domain. In the third step the user using the Session Initiation Protocol (SIP) [13] executes the IMS-AKA procedure for registration in the IMS domain.

The multi-pass AKA procedure involves a double execution of EAP-AKA and an execution of IMS-AKA that introduce an authentication overhead [17]. This overhead is related to: (i) the exchange of messages that cause delays in users' authentication (i.e., especially in cases that the users are located away from their home network) and consume radio resources; and (ii) the computational processing that will consume the limited energy and computational resources at the mobile devices. Therefore, the aforementioned multi-pass AKA procedure deteriorates the overall system performance and may impact negatively on the quality of service offered to the end-users.

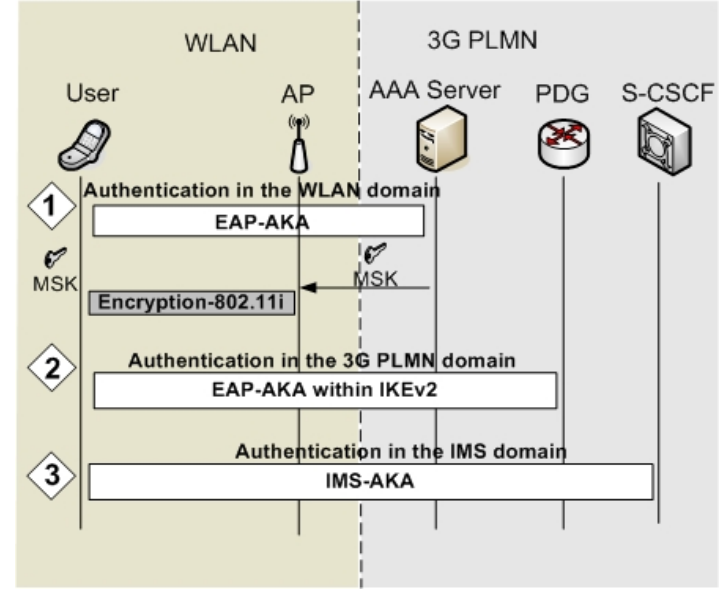

Figure 1: Multi-pass AKA Procedure for IMS services

There is a rather limited literature that copes with the aforementioned authentication overhead in NGN. L. Verti et al. [18] suggest an integrated authentication protocol, which is based on SIP and authenticates both the WLAN and the 3G PLMN within a single procedure, and thus reduces the overall authentication latency. However, the main drawback of this authentication procedure is that it is vulnerable to Denial of Service attacks. An adversary could simply send false authentication messages that the WLAN has to forward to the $3 \mathrm{G}$ PLMN causing overflow. N. Crespi et al. [19] propose the introduction of a new functional entity, called WLAN SIP proxy, in the WLAN that enables the latter to perform localized IMS services. However, this approach 
requires the implementation of the new entity and the related functionality increasing the deployment complexity.

This paper proposes a one-pass AKA procedure for next generation autonomous networks that reduces significantly the authentication overhead, compared to the multi-pass AKA procedure, without compromising the provided level of security. The proposed procedure first combines the initial and the second authentication steps by making a security key binding between them. This binding eliminates the need for execution of EAP-AKA for registration in the $3 \mathrm{G}$ PLMN. In the sequel, it combines the second and the third authentication steps by making a security identity binding between them. The second binding eliminates the need for execution of IMS-AKA for registration in the IMS domain, resulting in less exchange of messages and authentication computations. Apart from reducing the authentication overhead, the proposed procedure consumes less $3 \mathrm{G}$ authentication vectors, compared to the multi-pass AKA procedure. The one-pass AKA procedure has minimal impact on the network infrastructure and functionality, and does not require any changes to the existing EAP-AKA, IKEv2 and SIP protocols. A communication cost analysis is provided that estimates the cost improvement of the onepass AKA over the multi-pass AKA procedure.

The rest of this paper is organized as follows. Section 2 briefly presents the specified multi-pass AKA procedure. Section 3 describes and analyses the proposed one-pass AKA procedure. Section 4 evaluates the proposed procedure by elaborating a communication cost analysis. Finally, section 5 contains the conclusions.

\section{BACKGROUND}

\subsection{Multi-pass AKA Procedure}

Initial authentication for registration in the WLAN domain (step 1-Figure 1): The user and the WLAN are authenticated to each other using EAP-AKA [7] (see Figure 2). This authentication step involves the user, an Authentication, Authorization, Accounting (AAA) client that is actually a wireless Access Point (AP), and the AAA server (located at the service network) that obtains authentication information (i.e., $3 \mathrm{G}$ authentication vectors) from the Home Subscriber Server/Authentication Center (HSS/AuC) of the $3 \mathrm{G}$ PLMN where the user is subscribed, based on the user's permanent UMTS identity (i.e., International Mobile Subscriber Identity, (IMSI)). After executing EAP-AKA, the user and the AAA server share an EAP-AKA Master Key $(M K)$, which is used for the execution of EAP-AKA fast re-authentication and the generation of security keys [7]. The user and the AAA server use the $M K$ to calculate the Master Session Key $(M S K)$, and the second forwards it to the wireless AP. The AP and the user use this key to generate the WLAN session keys which are employed in the 802.11i security framework to provide security services [4]. After a successful EAP-AKA authentication, the user obtains a local IP address and can execute the IKEv2 protocol (i.e., next authentication step).

Second authentication for registration in the $3 G$ PLMN domain (step 2-Figure 1): In this step (see Figure 3) the user and an entity called Packet Data Gateway (PDG), which is located in the $3 \mathrm{G}$ PLMN, execute the IKEv2 protocol [8] that encapsulates EAPAKA for authenticating the user and the 3G PLMN. The PDG routes data traffic between a user and an external packet data network, which is selected based on the IMS services requested by the user. The IKEv2 protocol is executed in two phases (i.e., phase 1 and phase 2). In phase 1 the user and the PDG establish a bidirectional IKE Security Association (IKE SA) that protects all the subsequent IKEv2 messages (see Figure 3- step 1).

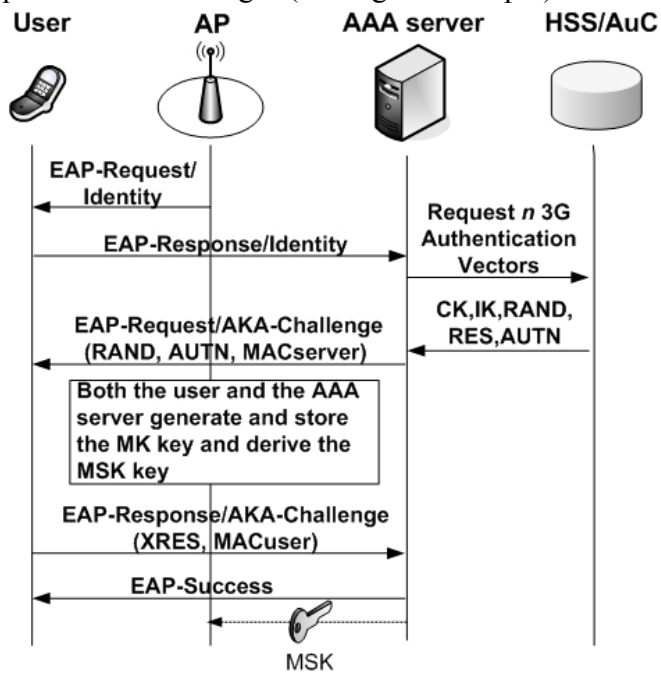

Figure 2: The EAP-AKA protocol (First Authentication Step)

In phase 2, the user and the AAA server execute EAP-AKA encapsulated in IKEv2 messages for mutual authentication. Note that the PDG forwards the EAP-AKA messages to the AAA server using the Diameter protocol [9]. In addition, the PDG is authenticated to the user using its certificate [2] (see Figure 3- step 3 ). At the end of this phase the user obtains from the PDG a global IP address, called Remote IP address, which is used for access to the IMS.

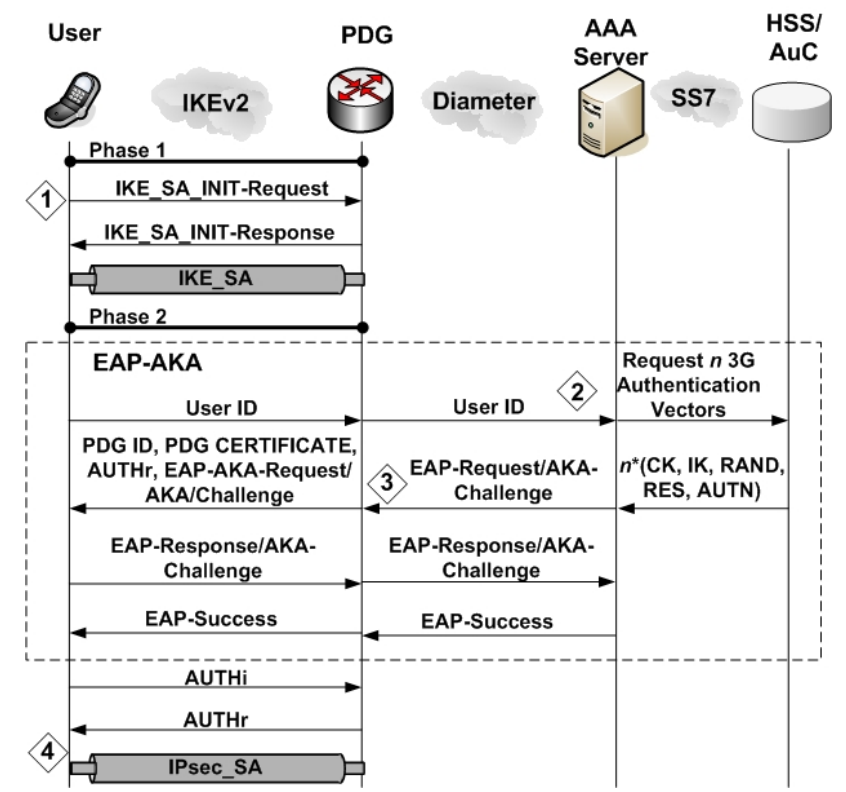

Figure 3: IKEv2 with EAP-AKA (second authentication step)

Moreover, an IPsec-based Virtual Private Network (VPN) tunnel [11] is deployed between the user and the PDG that uses the Encapsulating Security Payload (ESP) [12] protocol, which provides confidentiality and integrity to the data exchanged between them (see Figure 3 - step 4).

Third authentication for registration in the IMS domain (step 3Figure 1): In this step, the user and the IMS are authenticated to each other using the IMS-AKA procedure. In IMS, multimedia 
services are provided by the call session control functions (CSCF) using the SIP protocol. There are three types of CSCFs: (i) a proxyCSCF (P-CSCF) that is located in the visited network and is responsible for redirecting the SIP messages of users to their home networks; (ii) a serving-CSCF (S-CSCF) that is located in the home network of the user, communicates with the HSS and the AuC to receive IMS-related subscriber data and authentication information, and interacts with the application servers to obtain value added services; and (iii) an interrogating-CSCF (I-CSCF) that is responsible for selecting a S-CSCF for a user.

At the beginning of the IMS-AKA procedure, the user sends its permanent IMS identity (i.e., IP Multimedia Private Identity (IMPI)) to the S-CSCF via the PDG (see Figure 4). Based on the user's identity, the S-CSCF obtains $n 3 \mathrm{G}$ authentication vectors from the $\mathrm{HSS} / \mathrm{AuC}$. Note that a $3 \mathrm{G}$ authentication vector includes a random challenge (RAND), the authentication token (AUTN), the expected response (XRES), the encryption key $(C K)$ and the integrity key $(I K)$. In the sequel, the S-CSCF selects one out of the $n$ obtained authentication vectors to proceed with the IMS authentication procedure and stores the remaining $n$ - 1 for future use. Then, the S-CSCF sends the RAND and AUTN payloads to the user and the CK and IK keys to the P-CSCF. After receiving this information the user executes the UMTS-AKA algorithms, verifies the AUTN payload, generates the $I K$ and $C K$ keys, computes his response to the challenge (noted as an SRES payload), and sends it to the S-CSCF.

Upon receiving this message, the S-CSCF verifies the user's response to the challenge (SRES). If this check is successful, the SCSCF sends a success verification message to the user. Finalizing IMS-AKA, the user and the IMS network have been authenticated to each other, and the user and the P-CSCF share the CK and IK keys that provide confidentiality and integrity services respectively.

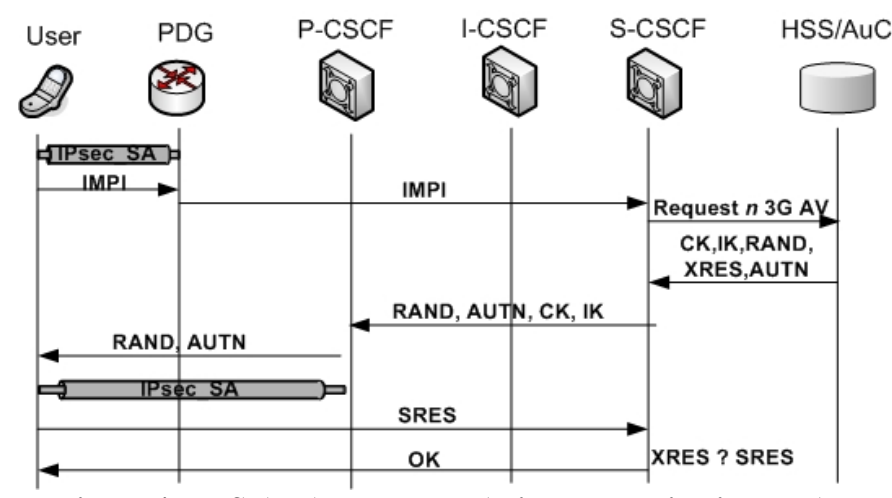

Figure 4: IMS-AKA procedure (third authentication step)

\section{ONE-PASS AKA PROCEDURE}

\subsection{Outline}

To address the multiple authentication overhead of the aforementioned multi-pass AKA procedure, this paper proposes a one-pass AKA procedure. The proposed procedure reduces the authentication traffic and the related computations, compared to the multi pass AKA procedure, without compromising the provided level of security. Similarly to the multi-pass AKA procedure, the one-pass AKA includes three distinct authentication steps. In the first step, the user and the WLAN are authenticated to each other performing EAP-AKA. In addition, in this step the user and the AAA server generate and store the $M K$ key, which is used in the second step. In the second authentication step, the user is authenticated to the 3G PLMN domain by executing pure IKEv2 that omits the encapsulation of EAP-AKA. In this step the authentication of the negotiating end-points (i.e., the user and the PDG) is based on the $M K$ key, which is generated in the first step (i.e., EAP-AKA). In this way, the one-pass AKA procedure achieves a security key binding between the first and the second authentication step. In the third authentication step, the one-pass AKA procedure authenticates the user by checking whether the IMPI identity and the IMSI identity belong to a legitimate user [14]. In this way, the one-pass AKA makes a security identity binding between the second and the third authentication step eliminating the need for executing the IMS-AKA for registration in the IMS domain.

The one-pass AKA procedure has minimal impact on the network infrastructure and functionality, and it does not require any changes to the existing EAP-AKA, IKEv2 and SIP protocols. However, the PDG must be able to modify the SIP messages as analyzed below. Another requirement is that the PDG must be capable of retrieving the $M K$ key, which is generated in the initial EAP-AKA authentication, from the AAA server. As mentioned in the specifications of EAP-AKA [7] and analyzed below, the AAA server stores the $M K$ key and maintains a list that associates the user's identities (i.e., permanent (IMSI) and temporary) with the relative $M K$ key. Thus, the PDG can retrieve the $M K$ key from the AAA server using the Diameter protocol. It is worth noting that there is a trusted relationship between the PDG and the AAA server, since there is a pre-established IPsec tunnel between them that protects the exchange of Diameter messages [9]. In addition, this tunnel protects the conveyance of the $M K$ key during the execution of the proposed one-pass AKA procedure.

\subsection{Authentication Procedure}

\subsubsection{Initial Authentication}

The initial authentication step of the proposed one-pass AKA (i.e., EAP-AKA authentication between the user and the AAA server) is the same with the one of the multi-pass (see Figure 2) and starts when the wireless AP requests the user's identity (EAP Request/identity message). The latter replies by sending to the AAA server an EAP Response/identity message, which contains either its permanent (i.e., IMSI) or a temporary identity in the format of Network Access Identifier (NAI). After obtaining the user's identity, the AAA server checks whether it possesses a fresh $3 \mathrm{G}$ authentication vector, stored from a previous authentication with the specific user. If not, the AAA server sends the user's IMSI to the HSS/AuC and obtains $n$ fresh $3 \mathrm{G}$ authentication vectors. Recall that a $3 \mathrm{G}$ authentication vector includes a random challenge (RAND), the authentication token (AUTN), the expected response (XRES), the encryption key $(C K)$ and the integrity key $(I K)$ [15]. The generation of authentication vectors is based on the pre-shared (between the user and the $3 \mathrm{G}$ network) secret key, $K$, which is assigned to the user when it is subscribed to the UMTS network. In the sequel, the AAA server selects one out of the $n$ obtained authentication vectors to proceed with the EAP-AKA authentication procedure and stores the remaining $n$ - 1 for future use. From the selected authentication vector it uses the keys $C K$ and $I K$ as well as the identity of the user to compute the EAP-AKA $M K$ (see eq.1). This key is used as a keying material to generate the Master Session Key (MSK).

$$
\mathrm{MK}=\operatorname{prf}(\text { Identity }|\mathrm{IK}| \mathrm{CK})
$$

As mentioned previously, the AAA server has to store the $M K$ key in order to execute the EAP-AKA fast re-authentication procedure [7]. Then, the AAA server calculates a Message Authentication 


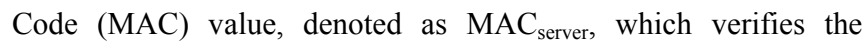
integrity of the next EAP-AKA message (i.e., EAP-Request/AKAChallenge). The AAA server sends the EAP-Request/AKAChallenge message to the user, which contains the RAND, AUTN and $\mathrm{MAC}_{\text {server }}$ payload. After receiving this information message, the user executes the UMTS-AKA algorithms and verifies the AUTN payload. Then, it generates the $I K$ and $C K$ keys, calculates the key $M K$, and produces the $M S K$ key. Likewise the AAA server, the user stores the generated $M K$ key in order to be able to execute a fast EAP-AKA re-authentication. If the verification of the $\mathrm{MAC}_{\text {server }}$ value is successful, the user computes its response to the challenge (noted as an SRES payload) and sends an EAPResponse/AKA-challenge message to the AAA server that includes the SRES and a MAC user value, which covers the whole EAP message.

Upon receiving the EAP-Response/AKA-challenge message, the AAA server verifies the received $\mathrm{MAC}_{\text {user }}$ value and checks if the received user's response to the challenge (SRES) matches with the expected response (XRES) of the selected $3 \mathrm{G}$ authentication vector. If all these checks are successful, the AAA server sends an EAPsuccess message along with the key MSK to the wireless AP. The latter stores the MSK key and forwards the EAP-success message to the user. Finalizing EAP-AKA, the followings have been achieved: (i) the user and the WLAN have been authenticated to each other; (ii) the user and the AAA server have stored the $M K$ key in order to be able to perform fast re-authentications; and (iii) the user and the wireless AP share the key MSK, which is employed in the 802.11i security framework for the generation of the WLAN session keys [4]. After a successful EAP-AKA authentication, the user obtains a local IP address and executes the IKEv2 protocol (i.e., next authentication step).

\subsubsection{Second Authentication Step}

In the second authentication step of the proposed procedure, the user and the $3 \mathrm{G}$ PLMN are authenticated using pure IKEv2. In addition, the user and the PDG establish a VPN tunnel that protects the data conveyed between them. The IKEv2 is executed in two phases (i.e., phase 1 and phase 2). In phase 1 the user and the PDG establish a bidirectional IKE_SA that protects all the subsequent IKEv2 messages. Note that the execution of this phase is the same with the one of the multi-pass AKA. To initiate the IKEv2 phase 1, the user sends to the PDG the SAil (message 1 - Figure 5) that denotes the set of cryptographic algorithms for the IKE_SA that it supports, the KEi that is the Diffie-Hellman value, and a $\mathrm{Ni}$ value that represents the nonce. The nonce is used as input to the cryptographic functions employed by IKEv2 to ensure liveliness of the keying material and protect against replay attacks. The PDG answers with a message (message 2- Figure 5) that contains its choice from the set of cryptographic algorithms for the IKE_SA (SAr1), its value to complete the Diffie-Hellman exchange ( $\overline{\mathrm{K} E r}$ ) and its nonce $(\mathrm{Nr})$. At this point, both the user and the PDG share a bidirectional IKE_SA that provides confidentiality and integrity services to the following IKEv2 messages.

After the establishment of the IKE_SA, the second step of the proposed one-pass AKA proceeds with the phase 2 of IKEv2, which authenticates the peers and establishes an IPsec SA. In contrast to the multi-pass AKA, which re-executes EAP-AKA and uses the certificate of the PDG to achieve mutual authentication between the user and the PDG, the proposed one-pass AKA omits these functions and the associated overhead. To accomplish mutual authentication both the user and the PDG calculate a hash value respectively (i.e., AUTHi and AUTHr payloads) using the $M K$ key, which is generated during the execution of EAP-AKA in the initial authentication step. Then, they send to each other the AUTHi and AUTHr payloads for verification, performing a security key binding between the initial authentication step (i.e., the execution of EAPAKA in the WLAN domain) and the second authentication step (i.e., the execution of IKEv2 in the 3G PLMN domain).

To initiate the IKEv2 phase 2, the user sends to the PDG a message that includes its identity, the SAi2 payload that contains the chosen cryptographic suit for the IPsec_SA that the user supports, the traffic selectors (TSi and TSr) that allow the peers to identify the packet flows that require processing by IPsec, and the Configuration Payload Request (CP-Request) that facilitates the user to obtain a Remote IP address from the PDG and get access to the IMS services. In addition, the user includes in this message the AUTHi payload, which is a MAC value over the first IKEv2 message (i.e., message 1 - Figure 5) using the stored $M K$ key. After receiving this information, the $\mathrm{PDG}$ forwards to the AAA server the user identity (IDi) including a parameter, which indicates that the authentication is being performed for access to the 3G PLMN [2]. This will facilitate the AAA server to distinguish between authentications for WLAN access or for 3G PLMN access. Based on the user's identity, the AAA server retrieves the appropriate $M K$ key and sends it to the PDG via the Diameter protocol (message 4 Figure 5). Recall that the MK key is conveyed in a secure manner, since there is a pre-established IPsec tunnel between the PDG and the AAA server.

Upon receiving the $M K$ key, the PDG verifies the AUTHi payload in order to authenticate the user. In the sequel, it generates the AUTHr payload by computing a MAC over the second IKEv2 message (i.e., message 2 in Figure 5) using the obtained $M K$ key, and sends it to the user. Except for the AUTHr payload, this message also includes the PDG's identity, the traffic selectors (TSi and TSr), the SAr2 payload that contains the chosen cryptographic suit for the IPsec_SA that the PDG supports, and the assigned user's Remote IP address that is included in the Configuration Payload Reply (CP-REPLY) payload. Finally, the user verifies the AUTHr payload using the $M K$ key and authenticates the PDG. At this point the authentication in the 3 G PLMN is completed and an IPsec_SA is established between the user and the PDG that provides security services to the transmitted data (see Figure 5).

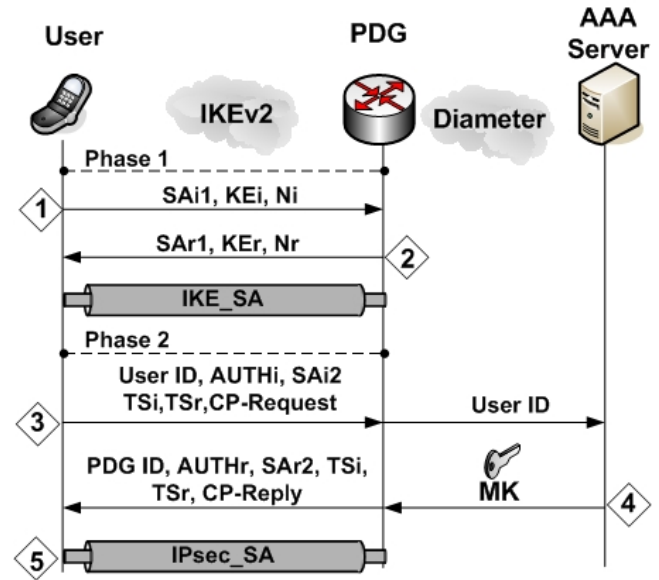

Figure 5: Second authentication step (one-pass AKA procedure)

\subsubsection{Third Authentication Step}

After the authentication in the 3G PLMN domain, the user proceeds to the third authentication step that registers it to the IMS domain (see Figure 6). In contrast to the multi-pass AKA, which performs 
mutual authentication between the user and the IMS, the one-pass AKA procedure authenticates only the user to the IMS network. The fact that the IMS is not authenticated to the user does not imply any security risk, since IMS is located within and operated by the 3 G PLMN, which has been already authenticated in the previous step.

In the third authentication step, the user first sends a SIP register message to the PDG that includes its IMPI identity using the previously established IPsec tunnel (Figure 6-step 1). Upon receiving the user's IMPI identity, the PDG retrieves the IMSI of the user by querying the Security Policy Database (SPD) of the IPsec protocol, which maintains the user's profile [11]. Then, the PDG incorporates the retrieved IMSI value of the user (i.e., imsi) in the SIP register message, and forwards it to the S-CSCF (Figure 6message 2). The latter stores the pair of identities (imsi, impi) in the user's record, and sends the impi value to the HSS/AuC (Figure 6message 3). Note that in case that the S-CSCF has already stored the pair of user's identities (imsi, impi) during a previous authentication, then the exchange of messages with the HSS/AuC (Figure 6-message 3) is omitted. Using the received IMPI value (i.e., impi), the HSS/AuC retrieves the IMSI identity of the user. We denote the retrieved IMSI value from the HSS/AuC as $\mathrm{IMSI}_{\mathrm{HSS}}($ impi $)$. In the sequel, the HSS/AuC stores the P-CSCF name and replies to the S-CSCF by sending the $\operatorname{IMSI}_{\mathrm{HSS}}($ impi) value. Finally, the S-CSCF checks whether the received and the retrieved IMSI values (i.e., imsi and $\operatorname{IMSI}_{\mathrm{HSS}}($ impi)) are the same. If $\mathrm{IMSI}_{\mathrm{HSS}}($ impi $)=i m s i$, then the S-CSCF sends a verification message to the user and the latter's registration in IMS is successfully completed (Figure 6-message 4). Otherwise, if $\operatorname{IMSI}_{\mathrm{Hss}}$ (impi) $\neq i m s i$, then the user is not valid and his registration to IMS is discarded.

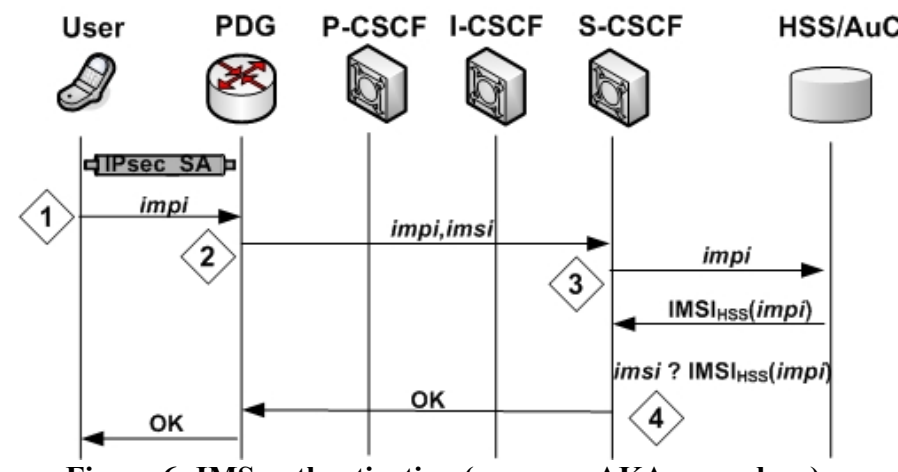

Figure 6: IMS authentication (one-pass AKA procedure)

\section{COST ANALYSIS}

Comparing the total number of messages required to complete the multi-pass AKA and the one-pass AKA procedure, it is evident that the proposed one-pass AKA reduces the authentication signaling and the associated overhead. More specifically, user registration in the 3G PLMN involves (see Figure 3): (i) the exchange of eight IKEv2 messages between the user and the PDG; (ii) the exchange of four Diameter messages between the PDG and the AAA server; and (iii) the exchange of two message between the AAA server and the $\mathrm{HSS} / \mathrm{AuC}$ for the retrieval of $3 \mathrm{G}$ authentication vectors. Thus, the multi-pass AKA procedure requires a total number of fourteen messages for a user's registration in the $3 \mathrm{G}$ PLMN.

On the other hand, for the same procedure the proposed one-pass AKA involves the exchange of four IKEv2 messages between the user and the PDG, and two Diameter messages between the PDG and the AAA server (see Figure 5). The AAA server does not exchange any credentials with the HSS/AuC, since the second authentication step of the proposed procedure avoids the retrieval of $3 \mathrm{G}$ authentication vectors. Thus, the one-pass AKA requires only a total of six messages for a user's registration in the $3 \mathrm{G}$ PLMN.

Table 1. The number of messages exchanged for user's registration in the3G PLMN using the multi-pass and one-pass AKA procedure

\begin{tabular}{lcc}
\hline \hline Communication link & Multi-pass $\boldsymbol{A K \boldsymbol { A }}$ & One-Pass $\boldsymbol{A K \boldsymbol { A }}$ \\
\hline \hline User-PDG & 8 & 4 \\
\hline PDG-AAA server & 4 & 2 \\
\hline AAA server-HSS/AuC & 2 & - \\
\hline Total & $\mathbf{1 4}$ & $\mathbf{6}$ \\
\hline
\end{tabular}

In addition, uses registration in the IMS domain under the multipass AKA involves (see Figure 4) the exchange of four SIP messages between the user and the P-CSCF, and the exchange of two messages between the P-CSCF and the HSS/AuC for the retrieval of $3 \mathrm{G}$ authentication vectors. Thus, the multi-pass AKA requires a total number of six messages for a user's registration in the IMS.

On the other hand, for the same procedure the proposed one-pass AKA involves (see Figure 6) the exchange of two SIP messages between the user and the P-CSCF, and two messages between the $\mathrm{P}-\mathrm{CSCF}$ and the HSS/AuC in case that the P-CSCF has not previously stored the IMSI of the user. Otherwise, the P-CSCF does not exchange any credentials with the HSS/AuC. Thus, the one-pass AKA procedure requires only a total of four or two messages for user registration in the IMS domain.

Table 2. The number of messages exchanged for user's registration in the IMS using the multi-pass and one-pass AKA procedure

\begin{tabular}{lcc}
\hline \hline Communication link & Multi-pass $\boldsymbol{A K \boldsymbol { A }}$ & One-Pass $\boldsymbol{A K \boldsymbol { A }}$ \\
\hline \hline User- S-CSCF & 4 & 2 \\
\hline S-CSCF-HSS/AuC & 2 & $-/ 2$ \\
\hline Total & $\mathbf{6}$ & $\mathbf{2 / 4}-$ \\
\hline
\end{tabular}

The reduced authentication overhead entails a reduced computational processing and energy cost at the level of mobile devices as well as a reduced consumption of the radio resources. Specifically, the mobile devices avoid the computational processing and the energy consumption induced by the execution of the UMTS-AKA, and the associated UMTS security algorithms. In addition, the reduced number of messages exchanged optimizes the usage of the radio resources improving the efficiency of user authentication in next generation mobile networks. Apart from reducing the authentication overhead, the one-pass AKA procedure consumes less $3 \mathrm{G}$ authentication vectors, compared to the multipass-pass AKA. Finally, the proposed procedure avoids the deployment of a public key infrastructure, since it does not employ certificates for the PDG authentication.

\section{CONCLUSIONS}

This paper proposes a one-pass AKA procedure for NGNs that reduces significantly the authentication overhead, compared to the multi-pass AKA procedure, without compromising the provided level of security. The proposed procedure first combines the initial and the second authentication steps by making a security key binding between them. The security key binding eliminates the need for execution of EAP-AKA for registration in the 3G PLMN. In the sequel, it combines the second and the third authentication steps by making a security identity binding between them. The 
security identity binding eliminates the need for execution of IMSAKA for registration in the IMS domain, resulting in less exchange of messages and authentication computations. Apart from reducing the authentication overhead, the proposed procedure consumes less $3 \mathrm{G}$ authentication vectors, compared to the multi-pass AKA procedure. Finally, the one-pass AKA procedure has minimal impact on the network infrastructure and functionality and does not require any changes to the existing EAP-AKA, IKEv2 and SIP protocols.

\section{ACKNOWLEDGMENTS}

Work supported in part by the project CASCADAS (IST-027807) funded by the FET Program of the European Commission and the 03ED910 research project, implemented within the framework of the Reinforcement Program of Human Research Manpower (PENED) and co-financed by National and Community Funds (75\% from E.U.-European Social Fund and 25\% from the Greek Ministry of Development-General Secretariat of Research and Technology)

\section{REFERENCES}

[1] 3GPP TS 23.234 (v7.3.0), "3GPP System to WLAN Interworking; System description”, Release 7, Sep. 2006.

[2] 3GPP TS 33.234 (v7.2.0), "3G security; WLAN interworking security; System description”, Release 7, Sep. 2006.

[3] 3GPP TS 23.228 (v8.1.0), "Technical Specification Group Services and Systems Aspects; IP Multimedia Subsystem Stage 2", Release 8, Jun 2007.

[4] 3GPP TS 33.203 (v7.6.0), "3G security; Access security for IP based services", Release 7, Jun. 2006.

[5] IEEE Std 802.11i, "Wireless Medium Access Control (MAC) and Physical Layer (PHY) Specifications: Medium Access Control (MAC) Security Enhancements", 2004.

[6] IEEE Std 802.11, "Wireless LAN Medium Access Control (MAC) and Physical Layer (PHY) Specifications", 1999.

[7] J. Arkko, H. Haverinen, "EAP-AKA Authentication", RFC 4187, Jan. 2006.
[8] C. Kaufman, "The Internet Key Exchange (IKEv2) Protocol", RFC 4306, Dec. 2005.

[9] P. Calhoun, J. Loughney, E. Guttman, G. Zorn, J. Arkko, "Diameter Base Protocol", RFC 3588, Sep. 2003.

[10] C. Laat, G. Gross, L. Gommans, J. Vollbrecht, D. Spence, "Generic AAA Architecture", RFC 2903, Aug. 2000.

[11] S. Kent, R. Atkinson, "Security Architecture for Internet Protocol", RFC 2401, Nov. 1998.

[12] S. Kent, R. Atkinson, "IP Encapsulating Security Payload (ESP)", RFC 2406, Nov. 1998.

[13] J. Rosenberg et al, "SIP: Session Initiation Protocol", RFC 3261, Jun 2002.

[14] Y.B. Lin, M.F. Chang, M.T. Hsu, L.Y. Wu, “One-pass GPRS and IMS Authentication Procedure for UMTS", IEEE Journal on Selected Areas in Communications, Vol. 23, No. 6, pp 1233-1239, Jun. 2005.

[15] C. Xenakis, L. Merakos, "Security in third Generation Mobile Networks", Computer Communications, Elsevier Science, Vol.27, No. 7, pp 638-650, May 2004.

[16] N. Asokan, V. Niemi, K. Nyberg. "Man-in-the-Middle in Tunneled Authentication Protocols". Lecture Notes in Computer Science, Vol. 3364, pp. 28-41, Springer 2005.

[17] C. Xenakis, C. Ntantogian, "Security Architectures for B3G Mobile Networks", Telecommunication Systems, Springer [In press, 2007].

[18] L. Veltri, S. Salsano, G. Martiniello, "Wireless LAN-3G Integration: Unified Mechanisms for Secure Authentication based on SIP", IEEE International Conference on Communications, (ICC), Istanbul, Turkey .2006.

[19] N Crespi, S. Lavaud, "WLAN Access to 3G Multimedia Services", Infromation and Communication Technologies, (ICT), Bangkok, Nov. 2004 